\title{
CONOCIMIENTO SOBRE ALIMENTACIÓN Y NUTRICIÓN DESPUÉS DEL DESARROLLO DE ACTIVIDADES DE EDUCACIÓN ALIMENTARIA ENTRE NIÑOS Y ADOLESCENTES DEPORTISTAS
}

\author{
Marina de Macedo Rodrigues Leite \\ Universidade Federal de Sergipe, Aracaju, Sergipe, Brasil \\ Ana Carolina Santos Barbosa Machado \\ Universidade Federal de Sergipe, Aracaju, Sergipe, Brasil \\ Danielle Góes da Silva \\ Universidade Federal de Sergipe, Aracaju, Sergipe, Brasil \\ Oscar Felipe Falcão Raposo \\ Universidade Federal de Sergipe, Aracaju, Sergipe, Brasil \\ Raquel Simões Mendes-Netto \\ Universidade Federal de Sergipe, Aracaju, Sergipe, Brasil
}

\begin{abstract}
Resumen
Objetivo: Evaluar el efecto de actividades de educación alimentaria en el conocimiento sobre nutrición básica y deportiva en niños y adolescentes deportistas. Métodos: participaron sujetos de ambos géneros, entre 9 y 19 años. Una muestra de 64 sujetos contestó al cuestionario antes del desarrollo de la intervención y los 27 sujetos que participaron de todas las actividades, volvieron a contestar al cuestionario después del desarrollo de la intervención. Para la evaluación de los cambios en su conocimiento sobre nutrición se aplicó el test de Wilcoxon. Resultados: Después de la intervención se observó un incremento significativo $(\mathrm{p}<0,05)$ en el porcentaje de aciertos para todos los temas del cuestionario (General, Nutrición Básica, Pirámide Alimentaria y Alimentación Deportiva).
\end{abstract}

Palabras-Clave: Educación Alimentaria y Nutricional. Conocimiento. Ejercicio. Niño. Adolescente.

\section{Financiación}

Para el desarrollo de este trabajo fueron concedidas becas en apoyo a la iniciación a la extensión universitaria, financiada por la Universidad Federal de Sergipe (PIBIX/UFS).

\section{Introducción}

Con frecuencia se observa una inadecuación en la ingesta alimentaria entre jóvenes. Entre los jóvenes brasileños, se caracteriza por una alta ingesta de galletas, en especial las galletas rellenas, los embutidos y bollería, asociado a una baja ingesta de legumbres, ensaladas y verduras, en comparación con los individuos adultos y mayores (IBGE, 2010). De manera semejante, se encontró en un estudio que evaluó la ingesta de adolescentes deportistas 
brasileños, una alta prevalencia de baja ingesta de raciones de los grupos de las frutas (83\%), las Verduras y Hortalizas (98\%) y las legumbres (72\%) (GONÇALVES; NOGUEIRA; DA COSTA 2014).

La estrategia Mundial sobre Régimen alimentario, actividad física y salud, indica la educación alimentaria y la utilización de las guías alimentarias como acciones de gran importancia para que se logren los objetivos de reducir la obesidad y otros factores de riesgo para el desarrollo de las enfermedades crónicas no transmisibles (WHO, 2004).

Durante la adolescencia, los jóvenes empiezan a asumir la responsabilidad por su comportamiento alimentario y de salud (TURCONI et al., 2008). De este modo, la educación alimentaria representa una importante ayuda a una mejor formación en cuanto a los hábitos alimentarios (WHO, 2004), una vez que entre adolescentes está influenciado en gran medida por la publicidad y grupo de amigos; Para ello, es necesario el desarrollo de programas educativos que permitan tener en cuenta la dimensión sociocultural de la alimentación, además de la biológica (BOOG, 2003).

Estudios que realizaron programas de educación alimentaria con niños (COSTA et al., 2009), y adolescentes deportistas (GONÇALVES; NOGUEIRA; DA COSTA, 2014) se mostraron eficaces al incrementar los niveles de conocimiento sobre el tema. Costa et al. demostraron un aumento significativo en el conocimiento en nutrición de niños sometidos a un programa de educación alimentaria en la escuela. Los autores destacan que el $76 \%$ de las cuestiones evaluadas obtuvieron resultados positivos después de la intervención.

Los programas de educación alimentaria destinados a atletas traen información sobre el conocimiento básico en nutrición y sobre alimentación deportiva, objetivan promover la salud y el rendimiento deportivo óptimo tras la corrección de hábitos alimentarios inadecuados (HEANEY et al., 2011).

El estudio de Jessri et al. (2010) evaluó el nivel de conocimiento en nutrición de jóvenes atletas y encontraron un alto porcentaje de respuestas erróneas, llegando a creer que los atletas adoptan las informaciones que obtienen de los medios de comunicación y de sus entrenadores como correctas. Pero, estas fuentes de información están más sujetas a errores, teniendo en cuenta que estudios que evaluaron el nivel de conocimiento de nutrición de entrenadores y técnicos, observaron un bajo conocimiento relacionado con la nutrición, incluyendo la nutrición deportiva (NASCIMENTO et al., 2013; COCKBURN et al., 2014).

Junto con los factores hereditarios y del entrenamiento, la adopción de una dieta adecuada es fundamental para que un deportista llegue a su mejor rendimiento (DAVAR, 2012). Actividades de educación alimentaria y nutricional pueden traer informaciones útiles a estos individuos, ayudándoles a hacer elecciones alimentarias que puedan actuar sobre la manutención de la salud y en la mejora de su rendimiento deportivo (GONÇALVES, 2009).

Con todo esto, el objetivo de este estudio fue evaluar el nivel de conocimiento en alimentación y nutrición básica y aplicada al deporte en niños y adolescentes deportistas, antes y después del desarrollo de actividades de intervención nutricional educativa.

\section{Material y métodos}

\section{Muestra}

Se trata de un estudio de intervención realizado con niños y adolescentes en edades comprendidas entre los 9 y 19 años, y edad media de 12,2 $(1,6)$ años, de ambos géneros ( chicos $=60,9 \%$ y chicas $=30,1 \%$ ) practicantes de natación y judo de centros deportivos de la ciudad de Aracaju, Sergipe, Brasil que participan de competiciones en nivel estadual y regional. El criterio para elegir los centros deportivos fue la autorización del centro considerando una viabilidad en cuanto a la participación de voluntarios y el interés de los 
mismos. Fueron incluidos en la búsqueda todos los niños y adolescentes que tuvieron participación autorizada por sus responsables, que firmaron el término de consentimiento libre y aclarado, conociendo todas las pautas a realizar. El proyecto fue aprobado por el Comitê de Ética e Pesquisa de la Universidad Federal de Sergipe con el número CAAE:0167.0.107.00009.

El proyecto fue desarrollado en dos fases, la primera fase consistió en una evaluación del conocimiento nutricional y alimentario de la población. Para ello, los 64 atletas, siendo 29 de natación y 25 de judo, contestaron individualmente un cuestionario de conocimientos sobre alimentación y nutrición.

La segunda fase del proyecto se dedicó al desarrollo de actividades educativas, con el objetivo de evaluar su efecto en el conocimiento sobre alimentación y nutrición de la población. Para la comparación de los resultados entre los periodos pre y post intervención, se consideró solamente los datos de los individuos que participaron de todas las acciones de educación alimentaria y nutricional, lo que representa un total de 27 sujetos.

\section{Actividades de educación alimentaria}

El proyecto se estructuró en tres etapas, dos acciones educativas con una duración de aproximadamente 40 minutos guiadas en un formato participativo y dinámico donde fueron planteadas cuestiones relevantes al tema, y estimulada la participación voluntaria. Y una tercera etapa en donde se les volvió a entregar el cuestionario. Las acciones ocurrieron en los propios centros deportivos, en días y horarios normales de entrenamiento.

\section{Desarrollo de la acción educativa}

Quince días después de la aplicación del cuestionario de conocimiento en alimentación y nutrición fue desarrollada una primera acción educativa, con el tema "Alimentación saludable y Pirámide Alimentaria". Esta acción tuvo el objetivo de aclarar la organización y disposición de los alimentos en la pirámide alimentaria y la importancia del consumo de cada grupo de alimentos, para un crecimiento y desarrollo saludables. Se utilizó un modelo de la pirámide alimentaria con alimentos sintéticos y rótulos de alimentos.

Al final de la acción los participantes deberían enseñar una comida saludable, teniendo en cuenta las explicaciones sobre la pirámide alimentaria, los grupos de alimentos y los principios de una alimentación saludable.

Al final de esta acción educativa, Se entregó a los participantes una cartilla que contenía de forma sencilla temas sobre alimentación y nutrición, como: pirámide alimentaria, grupos de alimentos e ingesta hídrica. Las informaciones de la cartilla fueron basadas en las orientaciones contenidas en la Pirámide Alimentaria Adaptada Para La Población Brasileña (PHILIPPI et al., 1999) y en la Guía Alimentaria Para La Población Brasileña (BRASIL, 2006).

La segunda acción educativa, realizada quince días después de la primera intervención, abordó el tema "Alimentación y entrenamiento deportivo" fue realizada con el objetivo de aclarar la relación entre alimentación y el rendimiento deportivo y estimular la aplicación de los conocimientos sobre alimentación y comidas saludables, pensando en el rendimiento deportivo, tanto en el entrenamiento como en las competiciones. Los recursos utilizados fueron alimentos sintéticos y rótulos de los alimentos.

Fueron destacados conceptos relacionados con el tiempo para alimentarse antes del ejercicio, enfatizando la importancia de una comida para garantizar el aporte energético para el entrenamiento y adecuación al tiempo disponible para digerir los alimentos. Después de exponer los conceptos iniciales fue pedido que tres grupos montasen lo que para ellos 
representaría una merienda ideal para el entrenamiento respetando los períodos de 1 hora, 30 minutos y 15 minutos antes del ejercicio.

Para el período durante el entrenamiento fue aclarado a los atletas la necesidad de líquido en intervalos regulares para evitar la deshidratación, debido al aumento de la temperatura corporal y perdida de líquido a través del sudor.

Para la fase post entrenamiento o competición fue abordada la necesidad de más una merienda, destacando que esta debe ser realizada lo más rápido posible. Fueron destacados los tipos de alimentos que deben componer esta merienda, y los atletas fueron invitados para demostrar lo que consumen usualmente después de un entrenamiento. En seguida fueron hechas sugestiones de meriendas adecuadas.

Al final de la acción educativa los participantes recibieron un tríptico que contenía informaciones sobre cómo alimentarse bien para el entrenamiento o competiciones.

Los 27 atletas que participaron de todas las actividades educativas además de la primera fase del proyecto, volvieron a contestar individualmente al mismo cuestionario de conocimiento en nutrición, con el objetivo de evaluar la modificación en el conocimiento en nutrición después del desarrollo de las actividades de educación alimentaria. La aplicación del cuestionario ocurrió cerca de 15 a 30 días después de la finalización de las actividades educativas, de acuerdo con la disponibilidad de los centros deportivos.

La pérdida de muestra, que fue de un $57,8 \%$ ocurrió debido al hecho de haber tenido en cuenta la comparación de los datos pre y post intervención únicamente de los jóvenes que participaron de las dos actividades de educación alimentaria y nutricional desarrolladas. Fueron excluidos aquellos individuos que no estaban presentes en el centro deportivo al momento del desarrollo de por lo menos una de las actividades. La razón principal para la exclusión de individuos fue la incompatibilidad entre los horarios de las actividades educativas y la disponibilidad de los jóvenes.

\section{El cuestionario}

Para la evaluación del conocimiento en nutrición de la población fue utilizado un cuestionario adaptado con base en cuestionarios utilizados en estudios previos(GONÇALVES, 2009; ZAWILA; STEIB; HOOGENBOOM, 2003), conteniendo los temas abordados en las actividades de educación alimentaria y nutricional educativas.

El cuestionario posee 14 cuestiones, divididas en tres secciones. La primera sección contiene tres cuestiones de elección múltiple referentes al conocimiento básico en nutrición. La segunda sección está compuesta por una cuestión referente a la pirámide alimentaria, la representación gráfica de la guía alimentaria para la población brasileña, y la tercera sección aborda el tema nutrición y actividad física y está compuesta por una cuestión conteniendo 10 afirmaciones, que los atletas deberían marcar "sí" caso concordasen con la afirmación hecha, "no", caso no concordasen con la afirmación o "no sé".

\section{Análisis estadístico}

Para el análisis estadístico fue utilizado el software SPSS, versión 19. 0 (SPSS Inc., Chicago, IL). Para validación del cuestionario utilizado, se verificó en un primer lugar la consistencia interna, tras la realización del coeficiente de fiabilidad de Alfa de Cronbach $(\alpha)$. Según Streiner (2003) este coeficiente puede ser determinado a partir de una aplicación del cuestionario; Evalúa la correlación entre los ítems de un mismo constructo y es útil para atestar la fiabilidad de las respuestas obtenidas tras la aplicación de cuestionarios(14). Su resultado varía desde 0,00 , que representa ausencia de fiabilidad, a 1,00 que representa una 
fiabilidad perfecta. Un valor mínimo de 0,70 fue recomendado por Rowland, Arkkelin y Crisler (1991) para determinar la consistencia de los ítems de un mismo grupo de preguntas.

Para determinar la validez discriminante del cuestionario, lo mismo fue aplicado entre 19 estudiantes del último año de nutrición y 16 atletas adolecentes que no participaron del estudio principal. De esta forma, para ser considerado valido, el cuestionario debería ser capaz de diferenciar los participantes en diferentes niveles de conocimiento. Para la comparación del porcentaje de aciertos del cuestionario entre estos dos grupos, se aplicó el test no paramétrico Mann-Whitney.

Para el análisis de los resultados obtenidos en el presente estudio fueron utilizadas medidas de tendencia central (media), dispersión (Desviación Estándar), y prevalencias (absoluta y relativa). Para comparación del porcentaje de aciertos del cuestionario entre la primera y segunda aplicación, fue utilizado el teste de Wilcoxon, considerando significancia estadística para $\mathrm{p}<0,05$.

\section{Resultados}

En relación a la validez del cuestionario, se encontró que el valor de la consistencia interna de las respuestas obtenidas en el cuestionario aplicado en el presente estudio fue de 0,76, representando fiabilidad aceptable, según Rowland (15). Como validez discriminante, fue observado un porcentaje de aciertos medio significativamente mayor entre los estudiantes del último año de nutrición $(97,4 \%)$, que entre los adolescentes atletas $(57 \%)$, considerando, por lo tanto, la validez discriminante del cuestionario utilizado para evaluar el conocimiento en nutrición de los deportistas participantes del presente estudio.

Participaron en la primera fase del estudio 64 niños y adolescentes de los cuales un $61 \%$ del género masculino, practicantes de natación $(n=29)$ y judo $(n=35)$, con edad media de 12,2 (1,6) años.

Para evaluar el porcentaje de aciertos, el cuestionario fue inicialmente analizado sin tener en cuenta la fragmentación de temas, determinando de esta manera el resultado del conocimiento general en nutrición (CG). Todo seguido el cuestionario fue dividido en las secciones siguientes: Nutrición Básica (NB), Pirámide Alimentaria (PA) y Alimentación Deportiva (AD) para evaluar el conocimiento alimentario, en cada una de las secciones específicas.

En la tabla 1 se presenta el porcentaje de aciertos en media (DE), mínimo y máximo, obtenidos en la aplicación del cuestionario en la fase pre-intervención; teniendo en cuenta el CG y las secciones: NB, PA y AD.

Tabla 1. Media, desviación Estándar, mínimo y máximo del porcentaje de aciertos del conocimiento alimentario (general y por secciones) antes de la intervención de niños y adolescentes deportistas.

\begin{tabular}{ccc}
\hline & Media (DE) & Min. - Máx. (\%) \\
\hline Conocimiento General & $59,9(15,4)$ & $19,2-92,3$ \\
Conocimiento por sección & & \\
Básico & $87,1(19,0)$ & $12,5-100,0$ \\
Pirámide Alimentaria & $19,9(27,4)$ & $0,0-87,5$ \\
Alimentación Deportiva & $67,2(21,2)$ & $20,0-100,0$ \\
\hline
\end{tabular}

\section{Conocimientos Específicos}

Nutrición Básica. En esta sección del cuestionario la población obtuvo un porcentaje medio de aciertos de un 87,7\%. La cuestión “¿Qué es una alimentación saludable?” fue 
contestada correctamente por el $95,3 \%$ de los individuos. Y por el contrario un $22 \%$ de la población total, no supo contestar o lo hizo incorrectamente en la cuestión "Complete la frase. El pan pertenece al mismo grupo de alimentos que..."

Pirámide alimentaria. Antes de contestar la cuestión para insertar los grupos de alimentos en la pirámide alimentaria, los atletas debieron contestar si la conocían. En la primera fase de la investigación, 53,1\% de la población refirieron no conocer la pirámide alimentaria. En esta sección del cuestionario, los atletas obtuvieron una media de aciertos de un 23,6\%. Los grupos de alimentos que se encuentran en la base y en la cúspide de la pirámide fueron los que presentaron mayor porcentaje de errores. Entre los individuos que refirieron conocer la pirámide alimentaria, 34,5\% contestaron la base de la pirámide con el grupo "cereales, pan, pastas", y el grupo "óleos y grasas" fue colocado en la base de la pirámide por $17,2 \%$ de la población. Los grupos de las frutas y de las verduras fueron colocados en la cúspide de la pirámide por un $20,7 \%$ y $27,5 \%$ de los atletas, respectivamente.

Alimentación Deportiva. En esta sección, la población obtuvo un porcentaje de aciertos en torno al 71,5\%. La cuestión "Se debe beber líquidos antes, durante y después de una competición" fue contestada correctamente por el $87,5 \%$ de la población. Por otra parte, la cuestión "Durante el entrenamiento sólo necesito beber agua cuando tengo sed" presentó el mayor porcentaje de respuestas erróneas, con un 46,9\% de la población.

\section{Intervención Nutricional Educativa}

La figura 1, presenta los datos de conocimiento en alimentación de los 27 individuos que participaron de todas las actividades de intervención nutricional educativa, antes y después del desarrollo de las dos acciones. Se observó en la fase post intervención, un incremento significativo $(\mathrm{p}<0,05)$ en el porcentaje de aciertos de la población, tanto en relación al conocimiento general del cuestionario como en los conocimientos específicos (NB, PA y AD).

De las ocho cuestiones que componen el tema Nutrición Básica, solamente tres cuestiones no presentaron $100 \%$ de aciertos después de la intervención alimentaria.

El tema referido a la pirámide alimentaria fue lo que obtuvo mayor diferencia en cuanto al porcentaje de aciertos. Después de la intervención 100\% de los participantes refirieron conocer la pirámide alimentaria. El 74\% de la población contestaron correctamente todos los grupos de alimentos presentes en la pirámide. Con relación a los grupos que componen la base y la cúspide de la pirámide, se observó el 100\% de aciertos en el grupo de las grasas, un $96,3 \%$ en el grupo de los dulces, bollerías, caramelos y pasteles y un $90 \%$ en el grupo de los cereales, pan y pastas.

En el tema Alimentación Deportiva, después de la intervención, el 93\% de los atletas contestaron correctamente al no concordar con la afirmación "Durante el entrenamiento sólo necesito beber agua cuando tengo sed". Ésta había sido la cuestión con mayor porcentaje de error entre los atletas antes de la intervención. Además, se destacó la cuestión "Es imposible vencer una competición sin utilizar suplementación alimentaria", que obtuvo una reducción, en cuanto al porcentaje de error, del $51,8 \%$ a un $22,2 \%$ tras haber realizado la intervención alimentaria educativa. 
Figura 1. Porcentaje de aciertos en el cuestionario de conocimiento en nutrición entre niños y adolescentes deportistas de la ciudad de Aracaju, Sergipe, Brasil, antes y después del desarrollo de actividades de educación alimentaria, 2011 - 2012 (n=27).

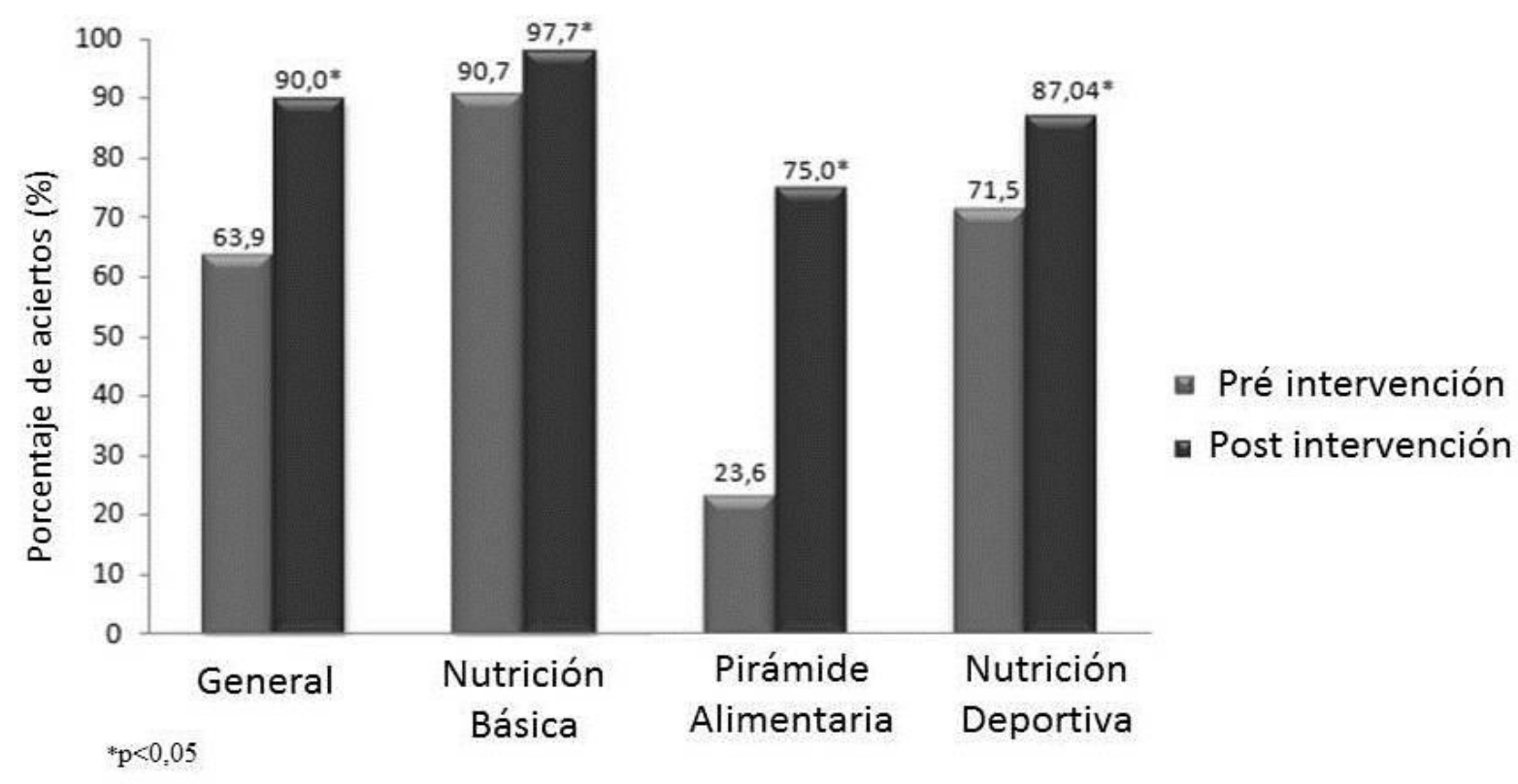

\section{Discusión}

La adopción de una dieta adecuada tiene un papel fundamental para un rendimiento deportivo óptimo (DAVAR, 2012). Para los jóvenes deportistas, el conocimiento sobre una alimentación y nutrición adecuada es esencial para la comprensión entre la asociación del binomio alimentación y rendimiento deportivo, dando lugar a actitudes alimentarias que favorezcan el rendimiento durante los entrenamientos y las competiciones (GONÇALVES, 2009).

En el presente estudio las actividades de educación alimentaria desarrolladas de forma sencilla fueron efectivas para elevar el nivel de conocimiento en nutrición de jóvenes atletas. El aumento del conocimiento alimentario después de la intervención fue significante, tanto en la variable que evaluaba el conocimiento general en alimentación como por cada tema específico (NB, PA y AD) abordado en el cuestionario y en las acciones educativas. El estudio de Valliant et al. (2012) realizó educación alimentaria individualizada con 11 jugadoras de vóley de la NCAA que resultó en una mejora en el conocimiento sobre nutrición y de la ingesta alimentaria de las atletas participantes.

En el periodo previo a la intervención alimentaria, a pesar de que los jóvenes deportistas presentaron un porcentaje de aciertos moderado sobre el conocimiento general (CG) (62,6\%), y un alto conocimiento en el tema Nutrición Básica (NB) (90,7\%), se observó una dificultad en cuanto a los alimentos que componen cada uno de los grupos alimentarios. Los atletas no asociaron los alimentos de acuerdo a su composición y función en el organismo, tuvieron como criterio la similitud entre ellos, como por ejemplo el origen (animal, vegetal) o la clasificación botánica en el caso de los vegetales.

Resultados similares, se observaron en otros estudios. El estudio de Turconi y cols. (2008) evaluó el conocimiento en nutrición de 532 adolescentes italianos practicantes de actividad física regular. El 49,2\% de los jóvenes presentaron conocimientos moderados sobre nutrición, y dificultad en temas específicos como en el caso de los nutrientes. 
Al igual que el estudio de Burkhart (2010), que evaluó el conocimiento sobre nutrición de 100 atletas adolescentes de niveles regional, nacional e internacional de Nueva Zelanda e identificó un conocimiento bajo en temas específicos como la composición de los alimentos.

La falta de conocimiento sobre el contenido de nutrientes de los alimentos, así como el aporte energético de los mismos dificulta la traducción del consejo alimentario a la hora de llevar a cabo elecciones alimentarias que cubran las necesidades energéticas y tengan mejor calidad nutricional (TURCONI et al., 2008).

La Pirámide Alimentaria (PA) fue definida con el objetivo de orientar a la población de forma clara y objetiva como poner en práctica las recomendaciones dietéticas (ACHTEMBERG et al., 1994) y fue adaptada para la población brasileña por PHILIPPI et al. (1999).

El estudio de Gonçalves, Nogueira y Da Costa (2014), utilizó la pirámide alimentaria como herramienta para educación alimentaria y demostró una mejoría en el conocimiento sobre nutrición y sobre la pirámide alimentaria en adolescentes deportistas, con o sin explicaciones adicionales de un profesional.

Por otro lado, algunos estudios demostraron una dificultad en el entendimiento de esta guía gráfica en forma de pirámide. En un estudio cualitativo Barbosa, Colares y Soares (2008) evaluaron la percepción de responsables por niños y recreadores en Brasil, a cerca de las guías gráficas infantiles, y encontró que en relación a la pirámide alimentaria ocurrió una idea de jerarquía entre los grupos alimentarios, que lleva al entendimiento de que los grupos de la cúspide son más importantes. Lobo y Martins (2014) resaltan la importancia de la presencia del nutricionista para aclarar las informaciones contenidas en la guía alimentaria en forma de pirámide, que sobre diversos aspectos puede tener su comprensión comprometida.

Así se concluye que la sencilla observación de la imagen de la pirámide alimentaria puede no fornecer todas las informaciones acerca de hábitos alimentarios saludables de manera clara y eficaz para los individuos, haciendo que sea necesario un contacto con profesional que aclare las informaciones contenidas en esta guía gráfica de la alimentación y aquellas que pueden ser inferidas a partir de una interpretación.

En el presente estudio la mayoría de la población $(53,1 \%)$ no conocía la pirámide alimentaria brasileña. Entre los individuos que refirieron conocer la pirámide, se observó un nivel de conocimiento bajo sobre este tema, que presentó un porcentaje de acierto de $23,6 \%$. Después del desarrollo de la intervención, la evaluación del conocimiento sobre la pirámide alimentaria presentó una mejoría significante en cuanto a los resultados.

El estudio de Gonçalves, Nogueira y Da Costa (2014) evaluó el conocimiento nutricional de 53 adolescentes practicantes de actividad física regular del Distrito Federal (Brasil) divididos en dos grupos (con y sin explicaciones del profesional), antes y después de actividades de educación alimentaria y se observó un aumento del conocimiento después de la intervención con relación al tema Pirámide Alimentaria en los dos grupos de intervención.

Además de la necesidad de aclarar algunas informaciones contenidas en la guía en forma de pirámide, se observa la necesidad de mayor divulgación de esto material, para que más personas conozcan y puedan ponerlo en práctica.

Sobre los conocimientos específicos en alimentación deportiva, se destacaron los temas relativos a la hidratación y suplementación alimentaria. Antes de la intervención los jóvenes atletas participantes de este estudio, reconocían la importancia del consumo hídrico, pero, creían que solamente necesitaban ingerir líquidos durante el entrenamiento si existiera la sensación de sed. Además, más de la mitad de la población creía que para vencer una prueba era necesario utilizar suplementación alimentaria.

Después de la intervención fue desmitificada la dependencia de la suplementación, resaltando la necesidad primaria de una alimentación adecuada. Los atletas además reconocieron la necesidad de ingestión hídrica anticipándose a la sensación de sed. 
Resultados similares acerca del conocimiento sobre hidratación y suplementación también fueron encontrados en el estudio de Amorim (2008) después de un programa de acciones educativas que abordaban el conocimiento en nutrición y actividad física, realizado con 55 atletas portugueses de fútbol con edades comprendidas entre 15 y 18 años.

Además de incrementar los conocimientos sobre alimentación y nutrición, las actividades de educación alimentaria son realizadas con el objetivo de presentar a los niños y adolescentes una alimentación saludable, para que adopten mejores hábitos alimentarios y los mantengan durante la adolescencia y la vida adulta.

Zancul y Valeta (2009) evaluaron la continuidad de la adopción de hábitos alimentarios saludables en estudiantes ocho meses después del término de la intervención alimentaria. Se volvió a aplicar el cuestionario utilizado durante el programa de educación alimentaria y se observó que un $67,6 \%$ de la población refirieron mantener el consumo de verduras crudas en la comida y cena, después del término de la intervención.

Davar (2012), llevó a cabo un estudio con 30 jugadoras de hockey universitarias de la India, con edades entre 17 y 23 años, en el cual todas las participantes consideraron que la nutrición es importante para deportistas, un 93,3\% consideró que la educación nutricional puede tener una influencia positiva sobre las elecciones alimentarias. Además, el mismo estudio identificó que las deportistas poseían interés en aprender sobre nutrición y un mismo porcentaje $(93,3 \%)$ consideró que el incremento en los conocimientos sobre nutrición representa la mejor manera de lograr cambios en la alimentación, siendo favorable a la adopción de unos hábitos alimentarios más saludables.

El pequeño tamaño de la muestra final del presente estudio representa una limitación. Pero el número final de individuos se asemeja con el observado en otros estudios de intervención nutricional educativa para niños, adolescentes y jóvenes deportistas realizados en Brasil (BOOG et al., 2003; COSTA et al., 2009; ZANCUL; VALETA, 2009;) o en otros países (VALLIANT et al 2012;).

A partir de estos resultados se puede afirmar que la educación alimentaria puede ser efectiva en el incremento del conocimiento en cuanto a una alimentación y nutrición adecuados para niños y adolescentes deportistas. Se sugiere la realización de nuevos estudios que puedan también evaluar el impacto de actividades educativas en alimentación y nutrición y los cambios en la alimentación a lo largo del tiempo. Además, se destaca la importancia de la presencia del nutricionista en centros deportivos, para que puedan orientar la población de jóvenes deportistas cuanto a la manera correcta de alimentarse, con el objetivo de mantener la salud, el desarrollo adecuado y rendimiento deportivo óptimo.

\title{
NUTRITIONAL KNOWLEDGE AFTER CONDUCTING NUTRITIONAL EDUCATION ACTIVITIES BETWEEN CHILDREN AND ADOLESCENTS SPORTSPEOPLE
}

\begin{abstract}
Objective: This study aimed to evaluate the effectiveness of food education activities on the basic and sports nutrition knowledge among young sportspeople. Methods: subjects of both genders, with ages between 9 and 19 years. To assess the nutrition knowledge, 64 subjects answered a questionnaire before the development of nutrition education activities. After the intervention, 27 subjects, who attended to all of the meetings, answered the same questionnaire. To assess the increase on their nutritional knowledge after the intervention the Wilcoxon test were used. Results: After the intervention, there was a significant increase in the percentage of correct answers $(\mathrm{p}<0.05)$ in all of questionnaire themes (General, Basic Nutrition, Food Pyramid and Sports Nutrition).
\end{abstract}


Keywords: Food and Nutrition Education. Knowledge. Exercise. Child. Adolescent.

\title{
CONHECIMENTO SOBRE ALIMENTAÇÃO E NUTRIÇÃO APÓS O DESENVOLVIMENTO DE ATIVIDADES DE EDUCAÇÃO ALIMENTAR ENTRE CRIANÇAS E ADOLESCENTES ESPORTISTAS
}

\begin{abstract}
Resumo
Objetivo: Avaliar o efeito de atividades de educação alimentar no conhecimento sobre nutrição básica e esportiva de crianças e adolescentes esportistas. Metodologia: participaram indivíduos de ambos gêneros, entre 9 e 19 anos. Uma amostra de 64 indivíduos respondeu ao questionário na fase pré-intervenção destes, 27 indivíduos participaram de todas as atividades e responderam ao questionário de conhecimento em nutrição após o protocolo de intervenção. Aplicou-se o teste de Wilcoxon para avaliação da mudança. Resultados: Após a intervenção, observou-se um aumento significativo $(\mathrm{p}<0,05)$ no percentual de acertos em todos os temas do questionário (Geral, Nutrição Básica, Pirâmide alimentar e Alimentação esportiva).

Palavras-chave: Educação Alimentar e Nutricional. Conhecimento. Exercício. Criança. Adolescente.
\end{abstract}

\section{Referências}

ACHTERBERG, G.; MCDONNELL, E.; BAGBY, R. How to put the food guide pyramid into pratice. J Amer Diet Ass. v. 94, n. 9, p. 1030-35, September, 1994.

AMORIM, S. F. Impacto da educação alimentar nos conhecimentos de nutrição e alimentação e na ingestão nutricional de jovens futebolistas. 2008. 85f. Dissertação Universidade do Porto. Porto, 2008.

BARbOSA, R. M. S.; COLARES, L. G. T., SOARES, E. A. Percepção de responsáveis e recreadores sobre diferentes representações gráficas de guia alimentar para crianças de dois a três anos. Rev Paul Pediatr. v. 26, n. 4, p. 350-6, 2008.

BOOG, M. C. F.; VIEIRA, C. M. OLIVEIRA, N. L.; FONSECA, O. L'ABBATE, S. Utilização de vídeo como estratégia de educação nutricional em adolescentes: "comer ... o fruto ou o produto?” Rev Nutr. v. 16, n. 3, p. 281-93, 2003.

BRASIL. Guia alimentar para a população brasileira: Promovendo a alimentação saudável. Brasília, 2005.

BURKHART, S. J. Assessment of nutritional knowledge and food skills in talented adolescent athletes. 2010. 305f. Dissertação (Master of Science in Human Nutrition) Massey University, Palmerston North, New Zeland, 2010.

COCKBURN, E.; FORTUNE, A.; BRIGGS, M.; RUMBOLD, P. Nutritional knowledge of UK coaches. Nutrients.; .v. 6, p.1442-53, 2014.

COSTA, A. G. M.; GONÇALVES, A.R.; SUART, D. A.; SUDA, G.; PIERNAS, P.; LOURENA, L. R; CORNACINI, M. C. M. Avaliação da influência da educação nutricional no hábito alimentar de crianças. Rev Inst Ciênc Saúde. v. 27, n.3, p. 237-43, 2009.

DAVAR V. Nutritional knowledge and attitudes towards healthy eating of college-going women hockey players. J Hum Ecol. v. 37, n. 2, p. 119-24, 2012. 
GONÇALVES, C. B. Consumo alimentar e entendimento da pirâmide alimentar adaptada em adolescentes fisicamente ativos do Distrito Federal. 2009. 154f. Dissertação (Mestrado em Educação Física) - Universidade de Brasília, Brasília, 2009.

GONÇALVES, C. B.; NOGUEIRA, J. A. D.; COSTA, T. H. M. The food pyramid adapted to physically active adolescentes as a nutrition education tool. Rev Bras Ciênc Esporte. Florianópolis, v. 36, n. 1, p. 29-44, jan./mar., 2014.

HEANEY, S.; O’CONNOR, H.; MICHAEL, S.; GIFFORD, J.; NAUGHTON G. Nutrition Knowledge: A Systematic Review. Int J Sport Nutr Exerc Metab. v. 21, p. 248-61, 2011.

INSTITUTO BRASILEIRO DE GEOGRAFIA E ESTATÍSTICA - IBGE. Pesquisa de Orçamentos Familiares 2008-2009: Análise do consumo alimentar pessoal no Brasil. Rio de Janeiro, 2011.

JESSRI, M.; JESSRI M.; RASHIDKHANI, B.; ZINN C. Evaluation of iranian college athletes' Sport nutrition knowledge. Int J Sport Nutr Exerc Metab. v. 20, p. 257-63, 2010.

LOBO, M.; MARTINS, I. Imagens em guias alimentares como recursos para a educação alimentar em aulas de ciências: Reflexões a partir de uma análise visual. Cad. Cedes, Campinas, v. 34, n. 92, p. 86-98, jan./Abr., 2014.

NASCIMENTO, M. V. S.; RAPOSO, O. F. F.; BRITO, C. J.; MENDES-NETTO, R. S. Conhecimento em nutrição de instrutores de musculação do município de Aracaju-SE. Rev Bras Ciênc Esporte. Florianópolis, v. 35, n.4, p. 1051-70, out./dez., 2013.

PHILIPPI, S. T.; LATTERZA, A. R.; CRUZ, A. T. R.; RIBEIRO, L. C. Pirâmide alimentar adaptada: guia para escolha dos alimentos. Rev Nutr. Campinas, v. 12, n. 1, p. 65-80, jan./abr., 1999 .

ROWLAND, D.; ARKKELIN, D.; CRISLER, L. Computer-based data analysis: using SPSSx iIn the social and Behavioral Sciences Chicago: Nelson-Hall; 1991.

STREINER, D. Starting at the beginning: an introduction to coefficient alpha and internal consistency. J Pers Assess. v. 80, n. 1, p. 99-103, 2003.

TURCONI, G.; GUARCELlO, M.; MACCARINI, L.; CIGNOLI, F.; SETTI, S.; BAZZANO, R. ROGGI, C. Eating habits and behaviors, physical activity, nutritional and food safety knowledge and beliefs in an adolescent Italian population. J Am Coll Nutr. v. 27, n. 1, p. $31-43,2008$.

VALLIANT, M. W.; EMPLAINCOURT, H. P.; WENZEL, R. K.; GARNER, B. H. Nutrition education by a registered dietitian improves dietary intake and nutrition knowledge of a NCAA female volleyball team. Nutrients. v. 4, p. 506-16, 2012.

WORLD HEALTH ORGANIZATION - WHO. Global Strategy on Diet, Physical Activity and Health. Genebra, 2004.

ZANCUL, M. S.; VALETA, L. N. Educação nutricional no ensino fundamental: resultados de um estudo de intervenção. Nutrire Rev Soc Bras Alim Nutr. São Paulo, v. 34, n. 3, p. 12540, dez., 2009.

ZAWILA, L. G.; STEIB, C. S. M.; HOOGENBOOM, B. The female collegiate cross-country runner: nutritional knowledge and attitudes. J Athletic Training. v. 38, n. 1, p. 67-74, 2003. 
Recebido em: 05/06/2015

Revisado em: 22/02/2016

Aprovado em: 22/02/2016

Endereço para correspondência:

raquelufs@gmail.com

Raquel Simões Mendes-Netto

Universidade Federal de Sergipe

Centro de Ciências Biológicas e da Saúde, Núcleo de Nutrição

Cidade Universitária

Jardim Rosa Elze

49100030 - São Cristóvão, SE - Brasil 